\section{A brief literature review on the management of post-haemorrhoidectomy pain}

\author{
Mohammed Mohsin Uzzaman, \\ Muhammed Rafay Sameem Siddiqui \\ Department of General Surgery, \\ Croydon Hospital, UK
}

\begin{abstract}
The most commonly encountered complication after haemorrhoidectomy is post-operative pain. Relief of this pain may aid earlier recovery. A literature search was performed examining the different surgical and medical agents for the relief of post haemorrhoidectomy pain using Pubmed, MEDLINE, EMBASE, CINAHL and Cochrane library databases. Pain can be relieved by surgical or medical agents. Surgery incorporates a risk of incontinence. A number of studies examine the role of medical agents.A variety of surgical techniques and medical agents are available to the clinician in the treatment of post haemorrhoidectomy pain. Tailored management to individual patients should ensure appropriate symptomatic control and prompt recovery.
\end{abstract}

\section{Introduction}

There are many surgical procedures such as the open haemorrhoidectomy of MilliganMorgan or Ferguson techniques. However there are other procedures that may be carried out with the aim of having low recurrence, minimal post-operative pain and early return to work. A recent systematic review by Burch comparing procedure for prolapse haemorrhoids (PPH) with conventional haemorrhoidectomy showed that PPH was associated with less pain in the immediate post-operative period, but a higher rate of recurrence. The relatively new technique of transanal hemorrhoidal dearterialization has been shown to cause low post-operative pain and have similar results to PPH. A recent systematic review concluded that it is a potential treatment option for second and third degree haemorrhoids. However, conventional open haemorrhoidectomy is still the most commonly performed operation for haemorrhoids

The most commonly encountered complication after haemorrhoidectomy is post-operative pain. Pain is usually most intense during the first passage of stools. Pain is the commonest reason for delayed patient discharge. Studies show that patients require 4 to 16 days to return to normal activity as a result of pain. Inadequate pain control also accounts for increased opioid requirements, post-operative nausea and vomiting, urinary retention and increased readmissions. As a result, the management of post-operative pain after haemorrhoidectomy is crucial both clinically and economically.

\section{Aetiology}

Haemorrhoids act as vascular cushions serving as a conformable plug to ensure complete closure of the anal canal and contribute approximately $15-20 \%$ of maximum resting pressure (MRP). ${ }^{1}$ MRP is mainly determined by anal sphincter function, while Mean Stretch Pressure (MSP) is entirely attributable to external anal sphincter. Ultra slow-waves activity (USWA) is the intrinsic contraction of smooth muscles. Studies have shown an elevation of MRP and ultra slow waves activity (USWA) in patients with haemorrhoids when compared with controls. ${ }^{2}$ This is due to abnormal, hypertonicity of the internal anal sphincter. It is not clear if the high resting anal pressures are caused by haemorrhoids or whether they are the cause of the haemorrhoids themselves.

Post-operative spasm of the internal anal sphincter is deemed to be a contributing factor in causing pain in patients with haemorrhoids. Several studies have shown that the pressures in the internal anal sphincter is greatest after a patient has had haemorrhoidectomy. ${ }^{2-4}$ This causes pain which leads to further increase in the pressure and so a vicious cycle ensues. There are other postulated mechanisms for experiencing post-operative pain after haemorrhoidectomy. One theory is that manipulation of the sensitive mucosa distal to the dentate line results in painful symptoms as a result of activating the stretch and somatic pain receptors. Poor and delayed wound healing will lead to epithelial denudation and will further exacerbate the symptoms. Another theory is that pain occurs due to incarceration of smooth muscle fibres and mucosa in the transfixed pedicle. The amount of pain experienced is dependant on a number of factors including the surgical technique, anaesthesia used, post-operative analgesia, early defecation of soft stools, adequate education, avoidance of dressings and subjective pain threshold.

PPH is associated with less post-operative pain. However, post-operative pain after PPH can also be caused by thrombosis of residual haemorrhoidal tissue, application of staples close to the anal verge, or incorporation of the rectal nerve/muscle in the suture. Some surgeons state that the closed Haemorrhoidectomy is more likely to cause pain due to incarcer-
Correspondence: Muhammed Rafay Sameem Siddiqui, Department of General Surgery Croydon Hospital, Croydon, UK.

Tel. +44.07890.726.471.

E-mail:md0u812a@zoho.com

Key words: haemorrhoids, pain control.

Received for publication: 10 September 2011. Accepted for publication: 21 October 2011.

This work is licensed under a Creative Commons Attribution NonCommercial 3.0 License (CC BYNC 3.0).

(C) Copyright M.M. Uzzaman, M.R.S Siddiqui, 2011 Licensee PAGEPress, Italy

Surgical Techniques Development 2011; 1:e32 doi:10.4081/std.2011.e32

ation of the smooth muscle fibres and mucosa in the transfixed pedicle. ${ }^{5}$ However, a recent meta-analysis comparing the open and closed techniques showed no difference in the maximum pain scores. ${ }^{6}$ In fact, they showed that closed Ferguson technique is associated with faster wound healing. They suggested that closed hemorrhoidectomy has theoretical advantages over the Milligan-Morgan technique by reducing the areas of denuded anal wall, which may reduce spasm and fissuring.

\section{Wound healing after haemorrhoidectomy}

Increased anodermal blood flow will help improve wound healing. Several studies done showed that improved wound healing occurs in response to reduced IAS pressure due to improved blood flow in patients with chronic anal fissure. ${ }^{7,8}$ As a result, patients experience less anal irritation, discharge and therefore post-operative pain. This is especially on defecation when most patients state that the pain is most severe. Improved wound healing also reduces the risk of secondary bleeding and anal structuring as there is less area of denuded wall. Also, good wound healing results in complete epithelisation of the affected region which reduces the risk of developing infections and dehiscence. Therefore, any sphincterotomy treatment which reduces spasm of IAS and increase anodermal blood flow will reduce the post-operative pain experienced and will improve wound healing.

\section{Surgical sphincterotomy}

Galizia et al. ${ }^{9}$ demonstrated reduced pain scores, lower analgesia requirements, and normalization of anorectal physiology in patients with elevated resting pressures before haemorrhoidectomy who underwent additional lateral sphincterotomy. Conversely, Khubchan- 
dani showed no significant benefit of an internal sphincterotomy in reducing post-operative haemorrhoidectomy pain. ${ }^{10}$

Faecal incontinence after a lateral sphincterotomy is due to damage to the sphincters and is usually permanent. This is the feared complication with lateral sphincterotomy, leading to a reduction in this form of treatment being administered and a search for a medical sphincerotomy to help with post-operative pain after haemorrhoidectomy.

\section{Simple analgesia}

A randomized trial using trimebutine, an anal sphincter relaxant, showed a reduction in anal resting pressure but no effect on either pain scores or analgesia requirements. ${ }^{11}$ Kilbride showed a satisfactory reduction in pain scores after using a transdermal fentanyl patch after haemorrhoidectomy. ${ }^{12}$ However, no further studies were done with either trimebutine or fentanyl patch so it is difficult to state whether such treatments are viable options in clinical practice. NSAIDs and opoids have been shown to be equally effective form of treatment in the post-operative management of haemorrhoids. Steroids are also used by some surgeons. A recent Randomised trial comparing betomethasone and diclofenac was conducted. The amount of analgesia used was less in the steroid group compared to the NSAID group leading to the suggestion that steroids are more effective analgesia for postoperative pain management in the haemorrhoidectomy patient. $^{13}$

\section{Laxatives}

As mentioned, the pain after haemorrhoidectomy is most intense after defecation. Several studies have shown that passing soft stools is associated with reduced pain..$^{14,15} \mathrm{As}$ a result, Laxatives such as lactulose are commonly prescribed after haemorrhoidectomy.

\section{Diltiazem}

Calcium channel blocking (CCBs) agents such as diltiazem and nifedipine have been used to block calcium uptake in the myocyte, thereby decreasing contraction of the IAS. Nifedipine was evaluated for treatment of anal fissure. With $0.2 \%$ nifedipine ointment, a mean reduction of $30 \%$ in MARP was observed. ${ }^{16}$ Oral diltiazem has also been shown to reduce the resting anal pressure. Carapeti $e t$ al. ${ }^{17}$ showed that a single dose of $60 \mathrm{mg}$ of diltiazem lowered MARP by a mean of $21 \%$. Another study by Carapeti et al. on patients with chronic anal fissure who were treated with $2 \%$ diltiazem for 8 weeks was conducted. ${ }^{18}$ The fissure healed in $67 \%$ of patients, there was a significant decrease in pain score after treatment with diltiazem $(\mathrm{P}=0.002)$ and MARP was significantly lowered $(\mathrm{P}=0.0001)$. Jonas et al showed that topical diltiazem was more superior to oral formulations in reducing MARP, promoting wound healing at 8 weeks, reducing pain and with less systemic sideeffects. ${ }^{19}$ A study conducted by Silverman et al conducted a randomised controlled trial assessing the efficacy of diltiazem on pain post-op haemorrhoidectomy. ${ }^{20}$ They applied either $\lg$ of $2 \%$ diltiazem ointment or a placebo ointment to matched controls by fingertip application to the perianal wound. They showed a significant reduction in perceived pain after haemorrhoidectomy and overall benefits with diltiazem ointment. There have been no further trials conducted in patients after a heamorhoidectomy. Consequently, although CCB are used widely for patients with anal fissures, more studies are needed before they are implemented in clinical practice for post-operative haemorrhoidectomy patients.

\section{Botolinium toxin}

The anaerobic bacterium Clostridium botulinum produces a family of toxins targeted to presynaptic nerve terminals. Studies of injection of botulinum toxin reported a significant decrease in anal resting pressure of 18$30 \%{ }^{21,22}$ When injected into the external anal sphincter, resolution of pain and ulcer healing occurred in $80 \%$ of cases of anal fissure. ${ }^{23}$ It enabled treatment of chronic uncomplicated anal fissure with increased sphincter tone with minimal risks of incontinence. Another study by Patti showed that treatment with botulinum toxin effectively decreased the MRP values together with a significant reduction in postoperative pain, both when resting and during defecation. They also showed an improvement in wound healing after haemorrhoidectomy with an earlier return to work and lower analgesic consumption. ${ }^{24}$

The most likely mechanism of action of botulinum toxin is through inhibition of acetylcholine release from presynaptic nerve endings, and of noradrenaline from sympathetic nerve endings. This results in a temporary block of neuromuscular transmission but not completely abolishing voluntary control. ${ }^{24}$ This is due to preferential block of the more active neurones. Weakness usually occurs after a few $\mathrm{h}$ with full return of muscle activity returns after the growth of new neuromuscular junctions within 3-4 months. Some have also suggested that botulinum toxin has an inhibitory effect on nociceptive neurones, and that it reduces the release of mediators associated with pain. ${ }^{25,26}$ Botulinum toxin has several potential advantages including single dose administration at the time of the operation, minimal side effect profile and a reversible long-lasting effect on the anal region.

\section{Local anaesthetic}

Some surgeons advocate local anaesthetic infiltration around the wound site. They advocate that this provides pain control immediately after an operation and during defecation when the patient will experience most pain. Moriasaki et al. ${ }^{27}$ and Ho et al. ${ }^{28}$ showed that LA infiltration reduced post-operative haemorrhoidectomy pain. However, the discomfort associated with the administration of local anaesthetic into the perineum limits its use, unless co-existing analgesia is used. There is also concern that this only provides short term pain-relief for 12-18 $\mathrm{h}$ and will subsequently other forms of analgesia will be needed in the long-term.

There may be a role for nerve block to the perineum in relieving post-operative pain. One of the concerns with this is that there is a risk of interrupting the micturation reflex and causing urinary retention. Imbelloni et al. demonstrated that bilateral pudendal nerve block with bupivicaine orientated by nerve stimulation resulted in adequate analgesia without the need for further analgesia and avoiding complications. ${ }^{28}$ They emphasised the importance of nerve stimulation as it localises the nerves more accurately and increases the rate success of nerve blockade. There is also increased length of nerve blockade with $20 \mathrm{~mL}$ of $0.25 \%$ percent bupivicaine providing a mean $23.8+/-4.8 \mathrm{~h}$ analgesia. Another study performed by Luck et al. reinforced this belief. ${ }^{29}$ The demonstrated a significant decrease in pain and analgesia requirements after hemorrhoidectomy after using a preemptive local anesthetic ischiorectal fossa blocks.

Sucralfate is composed of aluminium salt of sucrose octasulfate. It is a common anti-ulcer medication where it acts as a mechanical barrier as a result of the strong electrostatic interaction with proteins at the ulcer site. Other uses of sucralfate include treatment of radiation proctitis and keratoconjuctivitis. It has been shown to promote wound healing after second-degree burns. It is postulated that this occurs due to stimulation of fibroblast growth factor, stimulation of granulation tissues, antibacterial activity or angiogenic properties. ${ }^{30}$ Gupta et al. showed a significant reduction in pain after haemorrhoidectomy with topical application of 3 grams sucralfate and reduced analgesia requirements especially after 7 and 14 days..$^{30}$ They also showed a superior wound healing after application of sucralfate. They concluded that the effects were mediated by sucralfate binding to mucosal surfaces and interaction with surface phospholipids which stimulates prostaglandins and increases mucosal blood flow. The final result is reduced tissue oedema, flatter tissue margins, and faster epithelisation of the wound. The improved wound healing was also due to anti- 
bacterial actions of sucralfate. More research needs to done before this can be implemented clinically.

\section{Metronidazole}

A dose of metronidazole is commonly given after haemorrhoidectomy. Many have debated the role of regular metronidazole after haemorrhoidectomy. Carapeti et al. was the first study looking at the role of oral metronidazole on pain after day-case haemorrhoidectomy. ${ }^{31}$ Patients were either given $400 \mathrm{mg}$ tablets or placebo tds. They showed that pain was reduced every day in the first week but significantly between days 5-7. This also resulted in reduction in analgesia use, earlier return to normal activity and greater satisfaction in the metronidazole group. They went onto postulate that bacterial colonization and secondary infection to the post-operative anal wound leads to prolonged pain on days 3-7. This leads to sensitive friable anal mucosa due to inflammatory swelling and oedema. Metronidazole is an effective antibiotic against anaerobic bacteria and most likely to be effective against organisms that colonize anal wound. They postulated that open wounds are more likely to benefit from antibiotic use although closed wounds will also benefit from prophylaxis in order to prevent dehiscence. Further study by Al-Mulhim et al. showed that pain was significantly reduced every day of the week in the metronidazole group compared to the placebo group. ${ }^{32}$ They also showed earlier return to work but demonstrated no difference in complication rate. They agreed with carapetti group that oral metronidazole should be routinely administered after haemorrhoidectomy. Conversely, study by Balfour et al showed no difference between the group taking oral metronidazole and placebo group in terms of post-operative pain, time to first bowel movement, return to normal activity, complication rates and overall satisfaction. ${ }^{33}$

Two studies looked at the role of topical metronidazole on pain after haemorrhoidectomy. Nicholson and Armstrong showed that topical application of $2.5 \mathrm{cc} 10$ percent metronidazole was associated with reduced pain after 7 and 14 days. ${ }^{34}$ In addition, the metronidazole group was observed to have less postoperative oedema and significantly better healing at 2 weeks compared to the placebo group. Ala et al. similarly did a RCT comparing topical $10 \%$ metronidazole with placebo treatment. ${ }^{35}$ They showed that post-operative pain was less in the metronidazole group throughout the study period and also required less analgesia on days 2-7. They went onto conclude that metronidazole has 2 properties: an anti-inflammatory effect in the early phase and an antibacterial effect in the latter stages of treatment. Both phases will ultimately reduce post-operative pain. They also demonstrated reduced pain on defecation and suggested that inflammation was important in interfering with defecation post-surgery.

The emphasis is of preventing secondary infection in post-haemorrhoidectomy wounds. However, the incidence of perianal abcess, cellulitis and gangrene very rare after haemorrhoidectomy. Retrospective data report rates of $0-2 \%$ for abscess and fistula formation. ${ }^{36} \mathrm{~A} \mathrm{bac}$ teriological study of posthaemorrhoidectomy wounds for upto 4 weeks was conducted by De Paula et al. ${ }^{37}$ They demonstrated that all 20 wounds healed without problems and all were colonised by aerobic organisms. On the other hand, Brook and Frazier demonstrated that 18 of the 19 infected wounds after haemorrhoidectomy were infested with anaerobic organisms. $^{38}$ The most common anerobic organism isolated was Bacillus Fragilis and peptostrptoccoccus. It is based on findings like this that metronidazole - either oral or topical - is advocated as it is an effective antibiotic against anerobic organisms.

\section{GTN}

There is evidence that suggests that IAS is innervated by neurones that release nitric oxide (NO). Stimulation of these nerves results in the release of $\mathrm{NO}$ which then causes relaxation of the IAS. ${ }^{39}$ Exogenous application of nitrates has also been shown to have a similar response by relaxing the IAS. Organic nitrates include nitroglycerin which after cellular degradation results in the local release of NO. Loder et al. and Guillemot et al. showed that topical application of nitroglycerin results in the lowering of the IAS in normal human patients. ${ }^{40}$ It was thus popularly hypothesised that GTN and other nitrates can reduce pain by relaxing the IAS. Lund and Scholefield used $0.2 \%$ GTN ointment and showed that it decreased MARP by $33 \%$ and induced an increase in anodermal blood flow. ${ }^{41}$ Cundall $e t$ al. showed that there was a dose-response relationship for GTN therapy. ${ }^{42}$

Increasing doses from $0.1 \%$, to $0.2 \%$ to $0.4 \%$ GTN resulted in greater relaxation from $21.9 \%$ to $27.2 \% \%$ to $33.1 \%$ respectively.

GTN can also aid wound healing through an increase in anodermal blood flow secondary to a reduction in intra-anal pressure and vasodilatation of the arteriolar smooth muscles. ${ }^{7}$ It has been shown that GTN works most effectively on the upper two-thirds of the anal canal and to a lesser extent on the lower third of the anal canal [40]. Various trials have shown that topical GTN is effective in promoting healing in patients with chronic anal fissures ${ }^{43,44}$ As a result, GTN has superseded lateral sphincterotomy as the first line treatment for the management of chronic anal fissures.

One of the postulated drawback of GTN treatment is the short duration of action. Reports of up to 90 minutes have been report- ed in the literature. Relapse rates of $35 \%$ have been reported. This is why GTN ointment $(0.2 \%)$ must be applied around the anal opening 2-3 times daily, as well as before and after bowel movement. Because GTN requires frequent application, a GTN patch was investigated by Zuberi et al. to improve compliance and acceptability, and was found to be a suitable alternative. ${ }^{45}$ Isorsorbide mononitrate has been put forward as an alternative NO donor in the treatment of anal disorders. Unfortunately, ISMN seems to have very similar characteristics to GTN and is not yet deemed a suitable second-line agent. ${ }^{46}$ Coskun et al. used Nitroderm TTS band application after haemorrhoidectomy. ${ }^{47}$ They showed that the benefits of Nitraderm were greater in those patients who had pre-operative high resting anal pressures compared to matched placebo controls with a significant reduction in anal pressures and pain scores. There was very little benefit of Nitraderm TTS compared to placebo in those patients with normal pre-operative anal pressures.

One of the most feared complications with GTN treatment is the development of headaches. This is the commonest reason for poor compliance of treatment. The exact mechanism of GTN-induced headache is unclear. The most favored hypothesis is GTN-induced vasodilatation of the middle cerebral artery, mimicking a migraine attack. ${ }^{48,49}$ Those that favour topical GTN argue that the low doses are likely to promote local effects and unlikely to result in significant systemic complications. It must be remembered that $0.2 \%$ GTN was used in these trials which is a low dose of GTN. The mean amount patients were exposed is about $1 \mathrm{~g}$ per day. Jonas et al showed that topical application of GTN has a local effect on the internal anal sphincter and that there was no correlation between the plasma concentration and headaches. ${ }^{50}$ Lund and Kennedy and Carapetti et al. both suggested that higher doses such as $0.4 \%$ and $0.6 \%$ are likely to cause significant systemic effects with minimal added improvement in symptoms. ${ }^{43}$ The duration of GTN-induced headache is usually transient and do not usually interfere with treatment. The headaches usually encountered are minor, transient, self-limiting and can easily be treated with simple analgesia. Different formulations of GTN can have differing sideeffect profile. Coskun et al. introduced Nitroderm TTS 5 to the study. ${ }^{47}$ They argued that topical application of the conventional ointment three or four times a day is likely be absorbed rapidly by the anal skin and mucosa, causing a rapid surge in the plasma GTN levels. On the other hand, Nitroglycerin TTS 5 contains $25 \mathrm{mg}$ nitroglycerin, releasing 0.2 $\mathrm{mg} / \mathrm{h}$ continuously and resulting in exposure to $5 \mathrm{mg}$ over a $24 \mathrm{~h}$ period. It is rolled, with the nitroglycerin facing the outside, and then 
placed into the anal canal so allowing flatus to be passed without causing any disturbance to the patient. They demonstrated very few patients developing headaches and no patients required cessation of treatment with this form of formulation. Zuberi et al. used transdermal GTN patches where there was only a minor reduction in the incidence of headaches compared to GTN ointment. ${ }^{45}$

There have been several randomised-controlled trials that have looked at the role of GTN after haemorrhhoidectomy. It is difficult to draw on the overall benefits of GTN based on each study alone. A meta-analysis of the data will provide a more realistic impression on the overall benefits of GTN in patients with symptomatic grade III or grade IV haemorrhoids who undergo haemorrhoidectomy. As I mentioned, we used five out of the six papers for the meta-analysis to assess for the effects of GTN on pain scores. The other paper by Elton ${ }^{51}$ unfortunately did not assess for daily pain scores. Instead, they documented the average mean scores for each patient over the six-week study period. Despite this limitation, they showed that patients who had treatment with GTN ointment had an average pain score of 50.65 . This was less than the placebo group who had an average pain score of 73.50. Despite these differences, there was no statistical significance between the two groups.

In this study, I assessed the role of $0.2 \% \mathrm{GTN}$ in promoting wound healing three weeks after haemorrhoidectomy. I have performed a metaanalysis on three studies. I have shown that application of $0.2 \%$ GTN is associated with a significantly improved rate of wound healing at 3 weeks compared to matched placebo controls. Glyceryl trinitrate (GTN) act as nitric oxide donors and probably aid healing through an increase in local blood flow secondary to a reduction in intra-anal pressure.

It is worth noting that the overall reduction of pain in our study was most significant after day 3 and most significant on day 7 . Part of the analgesic effect of GTN is probably related to accelerated wound healing leading to reduced denuded epithelium. This is despite the fact that the rate of wound healing was similar in both groups.

Different formulations of GTN can have differing side-effect profile. Coskun et al. introduced Nitroderm TTS 5 to the study. ${ }^{47}$ They argued that topical application of

the conventional ointment three or four times a day is likely be absorbed rapidly by the anal skin and mucosa, causing a rapid surge in the plasma GTN levels. On the other hand, Nitroglycerin TTS 5 contains $25 \mathrm{mg}$ nitroglycerin, releasing $0.2 \mathrm{mg} / \mathrm{h}$ continuously and resulting in exposure to $5 \mathrm{mg}$ over a $24 \mathrm{~h}$ period. It is rolled, with the nitroglycerin facing the outside, and then placed into the anal canal so allowing flatus to be passed without causing any disturbance to the patient. They demonstrated very few patients developing headaches and no patients required cessation of treatment with this form of formulation. Zuberi et al. used transdermal GTN patches where there was only a minor reduction in the incidence of headaches compared to GTN ointment. ${ }^{45}$

Due to the risk of headaches, an alternative agent that will act as a sphincterotomy may be favoured as a first-line agent after haemorrhoidectomy. Sajid et al. conducted a metaanalysis comparing the effects of GTN and botulinium toxin on the management of chronic anal fissures. ${ }^{52}$ They showed that botolinium toxin was as effective as GTN in promoting wound healing without the risk of inducing headaches. Patti have conducted randomized controlled trials comparing application of 300 $\mathrm{mg}$ of $0.2 \% \mathrm{GTN}$ and $20 \mathrm{IU}$ intraoperative injection of Botonium toxin. ${ }^{53}$ They found that both agents effectively reduced MRP on days 5 and 40 whilst promoting similar wound healing rates. However, the Botox Group had much lower pain scores and reported no adverse effects such as headaches. Headaches only occurred in five out of fifteen cases in the GTN group but this was transient and relieved with oral nimesulide.

Another study by the same group combined small doses of GTN (100 mg $0.2 \%$ GTN tds for seven days) and intra-operative injection of 0.4 $\mathrm{mL} 20 \mathrm{UI}$ botonolinium toxin in ten patients undergoing grade III/IV haemorrhoidectomy. ${ }^{54}$ They showed effective reduction in MRP (days 5 and 40), reduced post-operative pain scores at rest and defecation with very minor sideeffect profile (1 case of headache).

GTN ointment is not statistically significant in reducing pain on post-operative Day 1. This is probably because the major contributor of pain in the early period post-operatively is due to trauma of surgery. This is particularly, due to the disturbance of the mucosa distal to dentate line. The pain caused by spasm of the internal anal sphincters becomes more important in the intermediate period (after 1 or 2 days).

Vita et al conducted a study using oral metronidazole, $0.2 \%$ GTN and lactulose in 15 patients compared to 15 patients on placebo treatment. $^{55}$ They demonstrated that the treatment group experienced significantly reduced pain scores on days $2 / 3 / 6 / 7$, reduced pain on the first two episode of defecation and reduced analgesic requirements compared to the placebo group. They attributed the low pain experienced on days 2 and 3 due to GTN-induced reduction of anal sphincter spasm and the significant benefits on days $6 / 7$ due to the effects of GTN/metronidazole in promoting wound healing and preventing secondary infection.

\section{Conclusions}

A variety of surgical techniques and medical agents are available to the clinician in the treatment of post haemorrhoidectomy pain. Tailored management to individual patients should ensure appropriate symptomatic control and prompt recovery.

\section{References}

1. Chauhan A, Thomas S, Bishnoi PK, Hadke NS. Randomized controlled trial to assess the role of raised anal pressures in the pathogenesis of symptomatic early hemorrhoids. Dig Surg 2007;24:28-32.

2. Patti R, Almasio PL, Arcara M, et al. Longterm manometric study of anal sphincter function after hemorrhoidectomy. Int $\mathbf{J}$ Colorectal Dis 2007;22:253-7.

3. Alper D, Ram E, Stein GY, Dreznik Z. Resting anal pressure following hemorrhoidectomy and lateral sphincterotomy. Dis Colon Rectum 2005;48:2080-4.

4. Chen HH. Anal manometric findings before and after hemorrhoidectomy: a preliminary report. Changgeng Yi Xue Za Zhi 1999;22:25-30.

5. Ganio E, Altomare DF, Milito G, et al. Longterm outcome of a multicentre randomized clinical trial of stapled haemorrhoidopexy versus Milligan-Morgan haemorrhoidectomy. Br J Surg 2007;94:1033-7.

6. Ho YH, Buettner PG. Open compared with closed haemorrhoidectomy: meta-analysis of randomized controlled trials. Tech Coloproctol 2007;11:135-43.

7. Kua KB, Kocher HM, Kelkar A, Patel AG. Effect of topical glyceryl trinitrate on anodermal blood flow in patients with chronic anal fissures. ANZ J Surg 2001;71:548-50.

8. Altomare DF, Rinaldi M, Milito G, et al. Glyceryl trinitrate for chronic anal fissure-healing or headache? Results of a multicenter, randomized, placebo-controled, double-blind trial. Dis Colon Rectum 2000;43:174-9; discussion 9-81.

9. Galizia G, Lieto E, Castellano P, et al. Lateral internal sphincterotomy together with haemorrhoidectomy for treatment of haemorrhoids: a randomised prospective study. Eur J Surg 2000;166:223-8.

10. Khubchandani IT. Internal sphincterotomy with hemorrhoidectomy does not relieve pain: a prospective, randomized study. Dis Colon Rectum 2002;45:1452-7.

11. Ho YH, Seow-Choen F, Low JY, et al. Randomized controlled trial of trimebutine (anal sphincter relaxant) for pain after haemorrhoidectomy. Br J Surg 1997;84: 377-9. 
12. Kilbride M, Morse M, Senagore A Transdermal fentanyl improves management of postoperative hemorrhoidectomy pain. Dis Colon Rectum 1994;37:1070-2.

13. Kisli E, Baser M, Guler 0, et al. Comparison of the analgesic effect of betamethasone and diclofenac potassium in the management of postoperative haemorrhoidectomy pain. Acta Chir Belg 2005;105:388-91.

14. Johnson CD, Budd J, Ward AJ. Laxatives after hemorrhoidectomy. Dis Colon Rectum 1987;30:780-1.

15. London NJ, BramLey PD, Windle R. Effect of four days of preoperative lactulose on posthaemorrhoidectomy pain: results of placebo controlled trial. Br Med J (Clin Res Ed) 1987;295:363-4

16. Staneva-Stoytcheva D, Venkova K. Effects of the calcium antagonists diltiazem, verapamil and nitrendipine on the contractile responses of guinea-pig isolated ileum to electrical stimulation or carbachol. J Pharm Pharmacol 1992;44:321-5.

17. Carapeti EA, Kamm MA, Evans BK, Phillips RK. Topical diltiazem and bethanechol decrease anal sphincter pressure without side effects. Gut 1999;45:719-22.

18. Carapeti EA, Kamm MA, Phillips RK. Topical diltiazem and bethanechol decrease anal sphincter pressure and heal anal fissures without side effects. Dis Colon Rectum 2000;43:1359-62.

19. Jonas M, Neal KR, Abercrombie JF, Scholefield JH. A randomized trial of oral vs. topical diltiazem for chronic anal fissures. Dis Colon Rectum 2001;44:1074-8.

20. Silverman R, Bendick PJ, Wasvary HJ. A randomized, prospective, double-blind, placebo-controlled trial of the effect of a calcium channel blocker ointment on pain after hemorrhoidectomy. Dis Colon Rectum 2005;48:1913-6.

21. Brisinda G, Maria G, Bentivoglio AR, et al. A comparison of injections of botulinum toxin and topical nitroglycerin ointment for the treatment of chronic anal fissure. $\mathrm{N}$ Engl J Med 1999;341:65-9.

22. Mason PF, Watkins MJ, Hall HS, Hall AW. The management of chronic fissure in-ano with botulinum toxin. J R Coll Surg Edinb 1996;41:235-8.

23. Jost WH. One hundred cases of anal fissure treated with botulin toxin: early and long-term results. Dis Colon Rectum 1997;40:1029-32.

24. Patti R, Almasio PL, Muggeo VM, et al. Improvement of wound healing after hemorrhoidectomy: a double-blind, randomized study of botulinum toxin injection. Dis Colon Rectum 2005;48:2173-9.

25. Gazerani P, Staahl C, Drewes AM, ArendtNielsen L. The effects of Botulinum Toxin type A on capsaicin-evoked pain, flare, and secondary hyperalgesia in an experimental human model of trigeminal sensitization. Pain 2006;122:315-25.

26. Aoki KR. Evidence for antinociceptive activity of botulinum toxin type A in pain management. Headache 2003;43 Suppl 1:S9-15.

27. Morisaki H, Masuda J, Fukushima K, et al. Wound infiltration with lidocaine prolongs postoperative analgesia after haemorrhoidectomy with spinal anaesthesia. Can J Anaesth 1996;43:914-8.

28. Ho KS, Eu KW, Heah SM, et al. Randomized clinical trial of haemorrhoidectomy under a mixture of local anaesthesia versus general anaesthesia. Br J Surg 2000;87:410-3.

29. Luck AJ, Hewett PJ. Ischiorectal fossa block decreases posthemorrhoidectomy pain: randomized, prospective, doubleblind clinical trial. Dis Colon Rectum 2000;43:142-5

30. Gupta PJ, Heda PS, Kalaskar S, Tamaskar VP. Topical sucralfate decreases pain after hemorrhoidectomy and improves healing: a randomized, blinded, controlled study. Dis Colon Rectum 2008;51:231-4.

31. Carapeti EA, Kamm MA, McDonald PJ, Phillips RK. Double-blind randomised controlled trial of effect of metronidazole on pain after day-case haemorrhoidectomy. Lancet 1998;351:169-72.

32. Al-Mulhim AS, Ali AM, Al-Masuod N, Alwahidi A. Post hemorrhoidectomy pain. A randomized controlled trial. Saudi Med J 2006;27:1538-41.

33. Balfour L, Stojkovic SG, Botterill ID, et al. A randomized, double-blind trial of the effect of metronidazole on pain after closed hemorrhoidectomy. Dis Colon Rectum 2002;45:1186-90; discussion 90-1.

34. Nicholson TJ, Armstrong D. Topical metronidazole ( 10 percent) decreases posthemorrhoidectomy pain and improves healing. Dis Colon Rectum 2004;47:711-6.

35. Ala S, Saeedi M, Eshghi F, Mirzabeygi P. Topical metronidazole can reduce pain after surgery and pain on defecation in postoperative hemorrhoidectomy. Dis Colon Rectum 2008;51:235-8.

36. Pescatori M, Gagliardi G. Postoperative complications after procedure for prolapsed hemorrhoids (PPH) and stapled transanal rectal resection (STARR) procedures. Tech Coloproctol 2008;12:7-19.

37. de Paula PR, Speranzini MB, Hamzagic $\mathrm{HC}$, et al. Bacteriology of the anal wound after open hemorrhoidectomy. Qualitative and quantitative analysis. Dis Colon Rectum 1991;34:664-9.

38. Brook I, Frazier EH. Aerobic and anaerobic microbiology of infected hemorrhoids. Am J Gastroenterol 1996;91:333-5.

39. Chakder S, Rattan S. Release of nitric oxide by activation of nonadrenergic noncholinergic neurons of internal anal sphincter. Am J Physiol 1993;264:G7-12.

40. Loder PB, Kamm MA, Nicholls RJ, Phillips RK. 'Reversible chemical sphincterotomy' by local application of glyceryl trinitrate. $\mathrm{Br}$ J Surg 1994;81:1386-9.

41. Lund JN, Scholefield JH. Glyceryl trinitrate is an effective treatment for anal fissure. Dis Colon Rectum 1997;40:468-70.

42. Cundall JD, Gunn J, Easterbrook JR, et al. The dose response of the internal anal sphincter to topical application of glyceryl trinitrate ointment. Colorectal Dis 2001;3:259-62.

43. Lund JN, Scholefield JH. A randomised, prospective, double-blind, placebo-controlled trial of glyceryl trinitrate ointment in treatment of anal fissure. Lancet 1997; 349:11-4.

44. Bielecki K, Kolodziejczak M. A prospective randomized trial of diltiazem and glyceryltrinitrate ointment in the treatment of chronic anal fissure. Colorectal Dis 2003;5:256-7.

45. Zuberi BF, Rajput MR, Abro H, Shaikh SA. A randomized trial of glyceryl trinitrate ointment and nitroglycerin patch in healing of anal fissures. Int $\mathbf{J}$ Colorectal Dis 2000;15:243-5.

46. Tankova L, Yoncheva K, Kovatchki D, Doytchinova I. Topical anal fissure treatment: placebo-controlled study of mononitrate and trinitrate therapies. Int $\mathrm{J}$ Colorectal Dis 2009;24:461-4.

47. Coskun A, Duzgun SA, Uzunkoy A, et al. Nitroderm TTS band application for pain after hemorrhoidectomy. Dis Colon Rectum 2001;44:680-5.

48. Hansen JM, Pedersen D, Larsen VA, et al. Magnetic resonance angiography shows dilatation of the middle cerebral artery after infusion of glyceryl trinitrate in healthy volunteers. Cephalalgia 2007;27: 118-27.

49. Moppett IK, Sherman RW, Wild MJ, et al. Effects of norepinephrine and glyceryl trinitrate on cerebral haemodynamics: transcranial Doppler study in healthy volunteers. Br J Anaesth 2008;100:240-4.

50. Jonas M, Barrett DA, Shaw PN, Scholefield JH. Systemic levels of glyceryl trinitrate following topical application to the anoderm do not correlate with the measured reduction in anal pressure. Br $\mathrm{J}$ Surg. 2001;88:1613-6.

51. Elton C, Sen P, Montgomery AC. Initial study to assess the effects of topical glyceryl trinitrate for pain after haemorrhoidectomy. Int J Surg Investig 2001;2: 353-7.

52. Sajid MS, Vijaynagar B, Desai M, et al. Botulinum toxin vs glyceryltrinitrate for the medical management of chronic anal 
fissure: a meta-analysis. Colorectal Dis 2008;10:541-6.

53. Patti R, Almasio PL, Arcara M, et al. Botulinum toxin vs. topical glyceryl trinitrate ointment for pain control in patients undergoing hemorrhoidectomy: a random- ized trial. Dis Colon Rectum 2006;49:17418.

54. Patti R, Angileri M, Migliore G, et al. [Effectiveness of contemporary injection of botulinum toxin and topical application of glyceryl trinitrate against postoperative pain after Milligan-Morgan haemorrhoidectomy]. Ann Ital Chir 2006;77:503-8.

55. Di Vita G, Patti R, Arcara M, et al. A painless treatment for patients undergoing Milligan-Morgan haemorrhoidectomy. Ann Ital Chir 2004;75:471-4; discussion 4-5. 